\title{
A Study of Children's Body Image and Depression in Korea
}

\author{
Eun Mi Kim ${ }^{1}$, Hye Seon Choi ${ }^{2}$ and Hye Young $\mathrm{Ahn}^{3 *}$ \\ ${ }^{1}$ Department of Nursing, Graduate School, Eulji University, Daejeon, Korea \\ ${ }^{2}$ Department of Nursing, College of Nursing, Woosuk University, Chonbuk, Korea \\ ${ }^{3}$ Department of Nursing, College of Nursing, Eulji University, Daejeon, Korea \\ ${ }^{1}$ kem5757@naver.com, ${ }^{2}$ hschoi@woosuk.ac.kr, ${ }^{3 *}$ ahanaya@eulji.ac.kr
}

\begin{abstract}
This study aimed to determine the association between CRE and depression by BMI and BIP and help school-age children do health management though good body image formation. The participants for the study were 240 school-age children in South Korea. The sample include 130 boys (58.8\%) and 91 girls (41.2\%) children, 10-13years old (mean age $=11.9, S D=0.86$ ). Data analysis was performed using SPSS 21.0 for descriptive statistics, $\chi^{2}$-test, ANOVA. In this study, many of the school-age children thought that they were obese and made lots of attempts to lose weight, though they had normal weight. And children in the BMI-N group were more vulnerable to CRE and scored higher for depression as they were more likely to think that they were fat than those in the BMI-U and $O$ groups. This result suggests the need to put greater emphasis on education to allow normal-weight children to develop correct BIP and eating habits. It is therefore necessary to allow children to recognize their subjective body image and help children with distorted BIP establish a good body image.
\end{abstract}

Keywords: Body Mass Index, Body Image, Restraint of Eating, Depression, Child

\section{Introduction}

\subsection{Need for Study}

Both the socio-cultural atmosphere with preference for a thin body and the influence of mass media that commercialize the beauty of the body are making lots of children and adolescents think of themselves as obese and form a negative body image. Such a negative body image has led to body dissatisfaction and the percentage of students dissatisfied with their body, regardless of gender, has been on the continuous increase for the past ten years $[1,2]$. A study in school-age children found that there were more children satisfied with their body weight $(39.1 \%)$ than those hoping to lose weight (47.1\%). $44.4 \%$ of the boys and $61 \%$ of the girls in the BMI-underweight group had wrong perception of their body as average or fat and $7.2 \%$ of the boys and $16.7 \%$ of the girls in the BMI-normal group thought of their body as fatter than the actual one [3].

A study in adolescents found that although $10.5 \%$ of the adolescents were in the BMI$\mathrm{U}$ group and $68.5 \%$ were in the BMI-N group, $51.8 \%$ preferred a thinner body than theirs and hoped to lose weight [4]. A study in 423 school-age children found that $71.8 \%$ of the children were making efforts to lose weight and that $28.3 \%$ were under restraint of eating in pursuit of weight loss [5].

Children who think that they are fat, though their weight is normal, are very likely to make inadequate attempts to lose weight due to the increase in social preference for a thin body, not as a means of preventing the perceived adverse effects of obesity on health. To lose weight, most of them don't take nutrition necessary for growth into account but use undesirable methods, such as excessive restraint of eating, medication for losing weight,

* Corresponding Author 
vomiting, and use of diuretics, which can injure their health [6-9]. Such a wrong attempt to lose weight can cause physical problems, including undernourishment, for school-age children who undergo drastic growth and development [10]. Poor nutrition can not only cause fatigue, flu symptoms, sleeping disorder, and problems in the skin but also serious health problems, such as overeating and eating disorders [11]. Wrong body image perception (BIP) and body dissatisfaction can negatively affect emotional development: people dissatisfied with their body image were more susceptible to emotional problems, such as depression and lower self-esteem, than those satisfied with their body image [12].

A study in school-age children found that children perceiving themselves as overweight put their values at lower levels and were more likely to experience depressive symptoms than those with no such perception [13]. In reality, people who think of themselves as overweight and take greater interest in body weight were more likely to develop psychological problems, such as depression, than obese ones [14, 15]. Continuous restraint of eating for the purpose of weight loss can cause the problem of depression as well as eating disorders [16]. A distorted image of their body also has negative physical and mental effects [17]. It is therefore necessary to let students with distorted BIP know the adverse effects of the wrong perception on health and give education about good body image formation and eating habits in schools, at home, and in communities with the objective of allowing children to make physically and mentally healthy growth $[11,18]$. In the process of human development, school age is characterized by drastic growth and development, the increased demand for nutrients, eating habit formation, physical development, and mental development, which involves self-consciousness and value establishment [7]. It is therefore necessary to conduct research on how the social phenomena of distorted BIP and indiscreet weight loss due to the preference to a thin body affect school-age children. While the recent research on BIP and restraint of eating, a method of weight control, has been conducted mainly in middle and high school girls and young female adults, little research has been conducted in school-age children $[8,19-25]$.

It is therefore necessary to examine the association between cognitive restraint of eating (CRE) and depression by the body mass index (BMI) and BIP with the aim of determining how wrong interest in body weight and appearance, which is on the social increase, affects school-age children, help them form a good body image, and identify basic data necessary for healthcare education and nursing interventions to prevent wrong attempts to lose weight and depression.

\subsection{Objective}

This study aimed to determine the association between CRE and depression by BMI and BIP and help school-age children do health management through good body image formation, and the specific objectives are as follows:

1) To identify the characteristics related to the body image of the subjects.

2) To determine the differences in CRE and depression by BMI and BIP of the subjects.

3) To determine the differences in CRE and depression by the BMI categories of the subjects.

\section{Methods}

\subsection{Design}

This is descriptive research to determine the differences in CRE and depression by BMI and BIP of school-age children. 


\subsection{Subjects}

The target population in this study was composed of school-age children, who were fourth- to sixth-graders, in South Korea and the proximal population was composed of school-age children, who were fourth- to sixth-graders, in Regions G and C. The number of subjects was based on the study in elementary school children [10] and the one in elementary school girls [7]. The G-Power 3.1 program revealed that these studies had an effect size of 0.26 and 0.21 , respectively. For ANOVA, a total of 171 children were selected-57 per group - with an effect size 0.24 , the significance level 0.05 , testability 0.8 , and the number of groups 3 , on the basis of the effect size for the two articles. Taking the dropout rate into account, questionnaires were distributed to and collected from a total of 240 children. The return rate of questionnaires was $100 \%$ and a total of 221 copies were analyzed, with the exception of 19 with no response.

\subsection{Instrument}

2.3.1. Body Mass Index (BMI): The body mass index (BMI) based on the measurements of body weight and height was used. It is calculated by dividing weight $(\mathrm{kg})$ by a square of height (m2) [26]. The researcher personally entered the subjects' weight and heightas measured in the 2013 Student Health Test-in cooperation with healthcare teachers. Percentile for BMI was estimated on the basis of BMI for children and adolescents, as disclosed by the Korean Pediatric Society (2007): $<15$ percentile was categorized into the BMI-U group, 15 to $<85$ percentile into the BMI-N group, 85 to $<95$ percentile into the BMI-overweight group, and $\geq 95$ percentile into the BMI-obese group. This study combined BMI-overweight and obese groups and obtained a total of 3 groups.

2.3.2. Body Image Perception (BIP): The Children's Body Image Scale (CBIS) developed by Truby et al. was used to measure BIP [27]. Seven pictures with the identical height and different levels of obesity were used to measure BIP. These pictures ranged from 1 for thinness to 7 for obesity. The level of BIP is determined by subtracting the number for the picture with the ideal body image desired by a child from that for the picture showing his/her body image. The children with a positive score for BIP perceived their body as fatter than the ideal one and were categorized into the BIP-obese group. The children with a negative score for BIP perceived their body as thinner than the ideal one and were categorized into the BIP-thin group. The children scoring 0 for BIP had their body image identical with the ideal one and were categorized into the BIP-normal group.

2.3.3. Cognitive Restraint of Eating (CRE): Cognitive restraint of eating (CRE) is an act of restraining eating due to strong intention and consciousness [28]. In this study, the eating behavior scale developed by Kim and Kim in the conceptual framework of three aspects of eating behavior (CRE, disinhibition, and hunger), as suggested by Stunkard and Messick [29], was used. This four-point scale was composed of a total of 32 items in 3 areas: CRE (restrain eating), disinhibition (restrain eating and then eat), and hunger (suffer from hunger). A higher score means a greater tendency to restrain eating for the purpose of losing weight. The original instrument developed for adolescents was revised and adapted to school-age children in cooperation with two professors of nursing, and preliminary research was conducted in three children from each of fourth to sixth grades to test content validity. At the time of developing this instrument, Cronbach's $\alpha=.94$ for reliability of all of the 32 items and Cronbach's $\alpha=.87$ for reliability of the CRE scale. A previous study only using a CRE scale in elementary school children (10) found that Cronbach's $\alpha=.81$ for reliability of the instrument. As for reliability of this scale in this study, Cronbach's $\alpha=.89$. 
2.3.4. Depression: The Children's Depression Inventory (CDI), which had been standardized by Cho et al. on the basis of the instrument developed by Kovacs et al., was used to measure children's depression [30,31]. The Korean version of CDI is composed of a total of 27 self-administered items in a scale of 0-2 points, with the total score ranging from 0 to 54 and with a higher score meaning a higher level of depression. At the time of development, Cronbach's $\alpha=.87$ for reliability of the instrument and Cronbach's $\alpha=.88$ in Cho et al. (30). As for reliability in this study, Cronbach's $\alpha=.90$.

\subsection{Data Collection}

\subsubsection{Approval by IRB and consent to survey}

- This research plan was reviewed and approved (EU 13-40) by IRB.

- Before the research, approval was obtained from the principal of the school interested.

- The school-aged children were asked to give a written consent before data collection through a survey.

- The school-aged children were asked to send a written consent form explaining the goal and contents of the research to their parents and collect a signed form.

2.4.2. Preliminary Research: The CRE scale developed for adolescents was revised and adapted to school-age children in cooperation with two professors of nursing. After the revision and adaptation, preliminary research was conducted using a structured questionnaire for data collection in 9 children -3 from each of fourth to sixth grades - in another elementary school before the main research. The preliminary research confirmed no difficulty in understanding the items.

2.4.3. Main Research: Data collection was performed in December 2013 and the principal, the class teachers at fourth to sixth grades, and the healthcare teacher were given explanation about the goal and purport of the study and were asked for assistance with data collection.

The researcher and the research assistant went into each of the classrooms in which the survey was conducted and gave explanation about the goal and purport of the study to the students. The students were let to know that they could withdraw from the study anytime and were asked to complete questionnaires, which were then collected. It took them 10-15 minutes to complete the questionnaire.

\subsection{Data Analysis}

Data analysis was performed using SPSS 21.0.

1) Frequency, percentage, and the mean and standard deviation were used to analyze the characteristics related to body images, CRE, and depression.

2) $\boldsymbol{X}^{2}$-test was used to analyze the association between BMI and BIP. BMI.

3) One-way ANOVA was used to analyze the differences in CRE and depression by

4) One-way ANOVA was used to analyze the differences in CRE and depression by BIP.

5) One-way ANOVA was used to analyze the differences in CRE and depression by BIP in each category of BMI.

\section{Results}

\subsection{Characteristics Related to Body Image}

The subjects' general characteristics and characteristics related to body images are as follows. The subjects in this study included 130 boys (58.8\%) and 91 girls (41.2\%) in 
fourth to sixth grades. 99 children $(44.8 \%)$ were in the fourth grade, $51(23.1 \%)$ in the fifth grade, and $71(32.1 \%)$ in the sixth grade; the mean age was $11.9 \pm 0.86$ years. The mean weight was $40.9 \pm 11.25 \mathrm{~kg}$ and the mean height was $144.7 \pm 8.47 \mathrm{~cm}$.

\subsection{Association between BMI Category and BIP Group}

In the BMI-U group, 224 children (42.1\%) thought that they were thin, $21(36.8 \%)$ thought that they had a normal body, and $12(21.1 \%)$ thought that they were obese. In the BMI-N group, 13 children (10.7\%) thought that they were thin, $39(32.0 \%)$ thought that they had a normal body, and $70(57.4 \%)$ thought that they were obese. In the BMI-O group, no child thought that they were thin, $3(7.1 \%)$ thought that they had a normal body, and $39(92.9 \%)$ thought that they were obese. Significant correlation was found between BMI and BIP $\left(\boldsymbol{\chi}^{2}=63.374, \mathrm{p}<.001\right)<$ Table 1.>.

Table 1. Relationship between BMI Groups and BIP Groups

\begin{tabular}{|c|c|c|c|c|c|}
\hline Categories & $\begin{array}{c}\text { BIP-T } \\
(n=37) \\
n(\%)\end{array}$ & $\begin{array}{c}\text { BIP-N } \\
(n=63) \\
n(\%)\end{array}$ & $\begin{array}{c}\text { BIP-O } \\
(n=121) \\
n(\%)\end{array}$ & $\chi^{2}$ & $p$ \\
\hline BMI-U & $24(42.1)$ & $21(36.8)$ & $12(21.1)$ & \multirow{3}{*}{63.374} & \multirow{3}{*}{$<.001$} \\
\hline BMI-N & 13(10.7) & $39(32.0)$ & $70(57.4)$ & & \\
\hline BMI-O & $0(0.0)$ & $3(7.1)$ & $39(92.9)$ & & \\
\hline
\end{tabular}

$\mathrm{BMI}=$ Body Mass Index, BIP= Body Image Perception,

BMI-U=BMI-Underweight, BMI-N=BMI-Normal, BMI-

$\mathrm{O}=\mathrm{BMI}$-Overweight/Obesity,

BIP-T= BIP-Thinness, BIP-N= BIP-Normal, BIP-O= BIP-

Obesity

\subsection{Differences in CRE and Depression by BMI}

Significant differences were found in the scores for CRE among the three BMI groups: 16.12 \pm 4.77 in the BMI-U group, 20.02 \pm 6.49 in the BMI-N group, and $24.45 \pm 6.05$ in the BMI-O group $(\mathrm{F}=23.358, \mathrm{p}<.001)$. The posttest found that the BMI-O group was at the highest level of restraint of eating, followed by the BMI-N group and the BMI-U group. No significant inter-group difference was found in the scores for depression by BMI of the subjects $<$ Table 2. $>$.

\subsection{Differences in CRE and Depression by BIP}

Significant differences were found in the scores for CRE among the three BIP groups: $16.70 \pm 4.73$ in the BIP-T group, 17.46 \pm 5.89 in BIP-N group, and 22.07 \pm 6.63 in the BIP-O group $(\mathrm{F}=17.496, \mathrm{p}<.001)$. The posttest found that the BIP-O group was at the highest level of CRE, followed by the BIP-N group and the BIP-T group.

Significant differences were found in the scores for depression among the three BIP groups: $8.03 \pm 5.82$ in the BIP-T group, $6.27 \pm 5.76$ in BIP-N group, and 9.62 \pm 7.50 in the BIP-O group $(\mathrm{F}=5.117, \mathrm{p}=.007)$. The posttest found that the BIP-O group scored highest for depression, followed by the BIP-T group and the BIP-N group<Table 2.>. 
Table 2. Comparison of CRE, Depression among BMI, BIP Groups

\begin{tabular}{|c|c|c|c|c|c|}
\hline Variables & Categories & $\mathrm{n}$ & $\mathrm{M} \pm \mathrm{SD}$ & $\mathrm{F}$ & $\begin{array}{c}P \\
\text { (Sheffe) }\end{array}$ \\
\hline \multirow{6}{*}{$\begin{array}{l}\text { Cognitive } \\
\text { Restraint of } \\
\text { Eating }\end{array}$} & $\mathrm{BMI}^{-\mathrm{U}^{\mathrm{a}}}$ & 57 & $16.12 \pm 4.77$ & \multirow{3}{*}{23.358} & \multirow{3}{*}{$\begin{array}{c}<.001 \\
(a<b<c)\end{array}$} \\
\hline & BMI-N $^{b}$ & 122 & $20.02 \pm 6.49$ & & \\
\hline & BMI-O $^{c}$ & 42 & $24.45 \pm 6.05$ & & \\
\hline & BIP-T ${ }^{a}$ & 37 & $16.70 \pm 4.73$ & \multirow{3}{*}{17.496} & \multirow{3}{*}{$\begin{array}{c}<.001 \\
(a<b<c)\end{array}$} \\
\hline & BIP-N ${ }^{b}$ & 63 & $17.46 \pm 5.89$ & & \\
\hline & $\mathrm{BIP}-\mathrm{O}^{\mathrm{c}}$ & 121 & $22.07 \pm 6.63$ & & \\
\hline \multirow{6}{*}{ Depression } & BMI-U & 57 & $8.40 \pm 6.24$ & \multirow{3}{*}{.313} & \multirow{3}{*}{.732} \\
\hline & BMI-N & 122 & $8.65 \pm 7.37$ & & \\
\hline & BMI-O & 42 & $7.67 \pm 6.47$ & & \\
\hline & BIP-T ${ }^{a}$ & 37 & $8.03 \pm 5.82$ & \multirow{3}{*}{5.117} & \multirow{3}{*}{$\begin{array}{c}.007 \\
(\mathrm{~b}<\mathrm{a}<\mathrm{c})\end{array}$} \\
\hline & BIP-N ${ }^{b}$ & 63 & $6.27 \pm 5.76$ & & \\
\hline & $\mathrm{BIP-O}^{\mathrm{c}}$ & 121 & $9.62 \pm 7.50$ & & \\
\hline
\end{tabular}

\subsection{Differences in CRE and Depression by BIP for each BMI Group}

In the BMI-N group, significant differences were found in the scores for CRE among the three BIP groups: $16.77 \pm 5.18$ in the BIP-T group, $18.21 \pm 5.91$ in BIP-N group, and $21.63 \pm 6.59$ in the BIP-O group $(\mathrm{F}=5.728, \mathrm{p}=.004)$. The posttest found that the BIP-O group was at the highest level of CRE, followed by the BIP-T group and the BIP-N group.

In the BMI-U and $\mathrm{O}$ groups, no significant difference was found in the scores for CRE among the three BIP groups. In the BMI-N group, significant differences were found in the scores for depression among the three BIP groups: $9.15 \pm 6.52$ in the BIP-T group, $5.33 \pm 4.85$ in BIP-N group, and $10.40 \pm 8.10$ in the BIP-O group $(\mathrm{F}=8.628, \mathrm{p}=.001)$. The posttest found that the BIP-O group was significantly more depressed than the BIP-N group.

In the BMI-U and $\mathrm{O}$ groups, no significant difference was found in the scores for depression among the three BIP groups < Table 3.>.

Table 3. Comparison of CRE, Depression among Different BIP Groups in each BMI Categories

\begin{tabular}{|c|c|c|c|c|c|c|}
\hline Variables & Category & $\begin{array}{l}\begin{array}{c}\text { BIP-Ta } \\
(\mathrm{n}=37) \\
\mathrm{M} \pm \text { SD }\end{array} \\
\end{array}$ & $\begin{array}{c}\text { BIP-Nb } \\
(n=63) \\
M \pm S D\end{array}$ & $\begin{array}{c}\text { BIP-Oc } \\
(n=121) \\
M \pm S D\end{array}$ & $\mathbf{t} / \mathbf{F}$ & $\begin{array}{c}P \\
\text { (Sheffe) }\end{array}$ \\
\hline \multirow{3}{*}{$\begin{array}{l}\text { Cognitive } \\
\text { Restraint of } \\
\text { Eating }\end{array}$} & BMI-U & $16.67 \pm 4.58$ & $14.62 \pm 3.83$ & $17.67 \pm 6.14$ & 1.886 & .162 \\
\hline & & & & & & $\begin{array}{c}.004 \\
(a, b<c)\end{array}$ \\
\hline & BMI-O & 0 & $27.67 \pm 3.51$ & $24.21 \pm 6.16$ & 0.955 & .345 \\
\hline \multirow{3}{*}{ Depression } & BMI-U & $7.42 \pm 5.45$ & $8.76 \pm 6.75$ & $9.75 \pm 6.98$ & 0.606 & .549 \\
\hline & BMI-N & $9.15 \pm 6.52$ & $5.33 \pm 4.85$ & $10.40 \pm 8.10$ & 8.628 & $\begin{array}{l}.001 \\
(\mathrm{~b}<\mathrm{c}) \\
\end{array}$ \\
\hline & BMI-O & 0 & $1.00 \pm 1.73$ & $8.18 \pm 6.42$ & -1.912 & .063 \\
\hline
\end{tabular}




\section{Discussion}

The problems related to the preference for a thin body have recently exerted negative effects on growth in childhood and adolescence and become important issues that schools and communities need to deal with. The finding that thin children with low BMI are more satisfied with their body than underweight or overweight ones [31] implies that children and adolescents regard a thin body as an ideal object of beauty.

It is therefore necessary to prevent school-age children from developing negative BIP, which results in the wrong methods of weight control and the psychological problems, including depression. This study aimed to examine BIP of school-age children by BMI and determine the association between CRE and depression.

\subsection{Association between BMI and BIP of Subjects}

As for BIP by BMI of the subjects, $21.1 \%$ in the BMI-U group, $57.4 \%$ in the BMI-N group, and $92.9 \%$ in the BMI-O group thought that they were obese. However, school-age children had seriously distorted BIP: $78.5 \%$ in the BMI-U or N group thought that they were obese. A study in elementary school children found that $82 \%$ of the subjects were dissatisfied with their body image and that $43 \%$ in the BMI-N group wanted themselves to be thinner [32]. A study in six- to eleven-year-olds obtained a supporting result that about a half of the children were dissatisfied with their body image and wanted themselves to be thinner [32]. The result that children fail to be satisfied with their body image like adolescents and adults and want to be thinner demonstrates that distorted BIP is not limited to adolescents and adults. The dissatisfaction with the body image can lead to the fear of weight gain and induce children to try to lose weight by using wrong methods, including excessive restraint of eating, which can adversely affect their growth.

School age and adolescence are characterized by drastic growth and development, which involves weight and body mass gain through normal growth and development. Children and adolescents can regard weight gain resulting from this normal growth and development as getting fatter and are very likely to try to lose weight in various ways. It is therefore necessary to help school-age children and adolescents understand a normal process of growth and development in those periods with the objective of preventing undesirable attempts to lose weight in which indiscreet restraint of eating blocks even nutrition necessary for growth.

\subsection{BMI, BIP, CRE, and Depression of Subjects}

The higher BMI, the higher score for CRE: 16.12 in the BMI-U group, 20.02 in the BMI-N group, and 24.45 in the BMI-O group. A study in which the identical instrument was used to determine CRE of fifth- and sixth-graders obtained a similar result: 19.59 in the BMI-U group, 21.23 in the BMI-N group, and 23.83 in the BMI-O group [10]. A study in seven- to twelve-year-old children also found that the higher BMI, the higher level of restraint of eating [33].

A study in which the same CRE scale as in this study was used in college students found that the BIP-O group scored highest for CRE, followed by the BIP-N group and the BIP-T group [21]. That is, the more likely they are to think of themselves as obese, the more likely they are to restrain eating. Such restraint of eating is affected by distorted BIP as well as by actual body weight and the distortion of BIP can be caused by exposure to mass media and social culture that prefer a thin body. A study in which dolls were used to determine the effects of exposure to mass media on distortion of BIP in children found that children exposed to a thin doll were at higher levels of restraint of eating and body dissatisfaction than those exposed to a normal-weight or obese doll. In contrast, children exposed to a normal-weight doll put no restriction on eating and had a feeling of happiness [34]. 
This result demonstrates that exposure to mass media strongly affects children's distortion of BIP. While exposure to mass media and the socio-cultural criteria do significantly affect the issue of distorted BIP for school-age children, their home, school, and peer group can distort their BIP [35]. To prevent this problem, therefore, it is necessary to allow children to have objective perception of their body, instead of evaluating it in comparison with models in mass media, and to identify the factors negatively affecting their BIP at home and in peer groups and make interventions. No significant difference was found in depression by the BMI categories of the subjects. As for differences in depression among the BMI categories, no significant difference was found among the BMI-U, N, and O groups.

A study applying the identical instrument in adolescents obtained a different result that the higher BMI, the higher score for depression: 10.2 in the BMI-N group, 10.3 in the BMI-overweight group, and 12.1 in the BMI-obese group [32]. Such a difference is probably because the psychological problem of depression is only affected by satisfaction with body weight and image and school-age children and adolescents have their depression affected by different factors.

A study in school-age children obtained an identical result that while obese children were at higher levels of body dissatisfaction and at lower levels of self-esteem than normal-weight and overweight ones, there was no significant difference in depression. Obese children dissatisfied with their body scored higher for depression than obese ones with no body dissatisfaction, who rather scored lower for depression than normal-weight ones [36].

This result demonstrates that children can have their psychological problems affected by dissatisfaction with their subjective body image, not by their actual body weight. School age is characterized by body image formation and negative BIP can prevent children from doing health behaviors for growth. It is therefore necessary to help children develop correct BIP in pursuit of their healthy growth.

As for the scores for depression in the BIP groups, the BIP-O group scored highest for depression, followed by the BIP-T group and the BIIP-N group. A study in elementary school children obtained the same result that children who thought that they were fat scored highest for depression [37]. A study in adolescents also obtained a supporting result that those who were more likely to think of themselves as fat and had greater fear of weight gain suffered from more serious depressive symptoms [24]. This study found that there was no significant difference in depression by BMI but the BIP-O group scored highest for depression. This result demonstrates that depression is more affected by a subjective body image than by the objective index, BMI. A study in children and adolescents also obtained a supporting result that negative self-esteem and depression were more affected by their subjective body image than by the actual degree of obesity [38]. School-age children's distorted perception that they are fat can cause psychological problems, such as depression, anxiety, and lower self-esteem, and wrong attempts to lose weight due to distorted BIP can cause them to experience depression [39]

In this study, every BMI and BIP group scored an average of $\leq 10$ for depression. Another South Korean study in which the same depression scale as in this study was used to measure depression in school-age children found that every BIP group scored $\geq 12$ for depression [37]. An overseas study found that every BMI group scored $\geq 10$ — which was higher than in this study-for depression. This result is probably because school-age children can have their depression strongly affected by the environments surrounding them, including family, peers, and school life. Since the children in this study scored low for depression, it is difficult to conclude that they are depressed due to distorted BIP. In making an approach to the problem of children's depression, therefore, it is necessary to address lots of their environmental factors as well as their subjective body image, body dissatisfaction status, and wrong attempts to lose weight. 
In this study [40], children in the BMI-N group were more vulnerable to CRE and scored higher for depression as they were more likely to think that they were fat than those in the BMI-U and O groups. This result suggests the need to put greater emphasis on education to allow normal-weight children to develop correct BIP and eating habits.

\section{Conclusion}

In this study, many of the school-age children thought that they were obese and made lots of attempts to lose weight, though they had normal weight. The children who had higher BMI and who were more likely to think that they were obese were more vulnerable to CRE. The children in the BIP-O group scored higher for depression, but no significant difference was found in depression among the BMI groups. This result demonstrates that children can have their depression more strongly affected by their subjective body image than by the objective index, BMI. It is therefore necessary to allow children to recognize their subjective body image and help children with distorted BIP establish a good body image.

It is also necessary to teach children with CRE some desirable methods of weight control, such as exercise, instead of restraint of eating that inhibits growth and development, and to manage their negative emotion, depression, and help them make physically and mentally healthy growth [40].

\section{References}

[1] A. Bair, J. R. Steele and J. S. Mills, "Do These Norms Make Me Look Fat? The Effect of Exposure to Others' Body Preferences on Personal Body Ideals”, Body Image., vol. 11, no. 3, (2014), pp. 275-281.

[2] M. M. Bucchianeri, A. J. Arikian, P. J. Hannan, M. E. Eisenberg and D. Neumark-Sztainer, "Body Dissatisfaction from Adolescence to Young Adulthood: Findings from a 10-year Longitudinal Study", Body Image., vol. 10, no. 1, (2013), pp. 1-7.

[3] J. O. Park, S. S. Jun, Y. H. Kim and S. H. Ahn, "Relationships between Sex and Perception of Body Shpape, Satisfaction with Body Weight and Exeriences of Weight Control according to Obesity Level among Elementary Schoolers", J Korean Soc Matern Child Health., vol. 9, no. 1, (2005), pp. 63-71.

[4] C. M. Lai, K. K. Mak, J. S. Pang, S. S. M. Fong, R. C. M. Ho and G. S. Guldan, "The Associations of Sociocultural Attitudes towards Appearance with Body Dissatisfaction and Eating Behaviors in Hong Kong Adolescents", Eating Behaviors., vol. 14, no. 3, (2013), pp. 320-324.

[5] S. H. Moon, "Gender Differences in Eating Disorders and Risk Factors in Upper Primary School Children”, J Korean Acad Child Health Nurs., vol. 14, no. 4, (2008), pp. 361-369.

[6] M. A. Doo and Y. H. Kim, "Recognition of Body Weight Loss according to Age and Gender", Korean J Nurt., vol. 40, no. 7, (2007), pp. 658-666.

[7] H. Sung, "Relationship between Eating Disorders, Physical Symptoms, Depression and Health Locus of Control among Elementary School Girls in South Korea", J Korean Acad Nurs., vol. 34, no. 3, (2004), pp. 576-585.

[8] S. Ursoniu, S. Putnoky and B. Vlaicu, "Body Weight Perception Among High School Students and Its Influence on Weight Management Behaviors in Normal Weight Students: A Cross-Sectional Study", Wiener Klinische Wochenschrift., vol. 123, no. 11-12, (2011), pp. 327-333.

[9] M. I. Jongenelis, S. M. Byrne and S. Pettigrew, "Self-Objectification, Body Image Disturbance, and Eating Disorder Symptoms in Young Australian Children”, Body Image., vol. 11, no. 3, (2014), pp. 290302.

[10] B. H. Kim and J. H. Choi, "Perceived Body Image, Intentional Caloric Restriction and Physical Manifestations of Unbalanced Nutrition according to Body Mass Index in Fifth and Sixth Grade Elementary School Students", Korean Acad Child Health Nurs., vol. 15, no. 4, (2009), pp. 259-366.

[11] M. H. Sung, "Relationships between BMI, Eating Disorders, Physical Symptoms and Self Esteem among Fifth Grade and Sixth Grade in an Elementary School Girls”, J Korean Acad Child Health Nurs., vol. 11 , no. 3, (2005), pp. 282-289.

[12] S. J. Paxton, D. Neumark-Sztainer, P. J. Hannan and M. E. Eisenberg, "Body Dissatisfaction Prospectively Predicts Depressive Mood and Low Self-Esteem in Adolescent Girls and Boys", Journal of Clinical Child \& Adolescent Psychology., vol. 35, no. 4, (2006), pp. 539-549.

[13] Y. M. Lee, "The Effect of Children's Perception of Body Shape and Body Image on Their Negative Emotions", Family and Environment Research, vol. 42, no. 8, (2004), pp. 133-145.

[14] O. S. Kim and K. H. Kim, "Weight, Self-Esteem, and Depression in High School and College Females", Korean Journal of Adult Nursing, vol. 12, no. 3, (2000), pp. 396-406. 
[15] W. H. Ting, C. Y. Huang, Y. K. Tu and K. L. Chien, "Association between Weight Status and Depressive Symptoms in Adolescents: Role of Weight Perception, Weight Concern, and Dietary Restraint”, Eur J Pediatr, vol. 171, no. 8, (2012), pp. 1247-1255.

[16] D. Neumark-Sztainer, M. Wall, N. I. Larson, M. E. Eisenberg and K. Loth, "Dieting and Disordered Eating Behaviors from Adolescence to Young Adulthood: Findings from a 10-year Longitudinal study", Journal of the American Dietetic Association, vol. 111, no. 7, (2011), pp. 1004-1011.

[17] D. S. Kim, H. S. Kim, Y. Cho and S. I. Cho, "The Effects of Actual and Perceived Body Weight on Unhealthy Weight Control Behaviors and Depressed Mood among Adult Women in Seoul, Korea", Journal of preventive medicine and public health, vol. 41, no. 5, (2008), pp. 323-330.

[18] H. K. Ro and J. Park, "Grade and Gender Differences in Dietary Behavior , Food Preference and Perception about Body Image of 4, 5 and 6th Grade Students in Elementary School", Korean J Dietary Culture, vol. 16, no. 2, (2001), pp. 158-169.

[19] S. K. Chaung, "Weight Control Practices, Body Image, Self Esteem and Depression accroding to Weight Perception of Adolescent Girls", Semyung University, vol. 7, no. -, (1998), pp. 619-632.

[20] S. K. Chaung, "Weight Control Practices and Body Image of Female College Students", Korean Journal of Health Education and Promotion, vol. 18, no. 3, (2001), pp. 163-175.

[21] Y. L. Ham and M. Park, "Effect of Type of Body Shape Perception on Health Concern, Depression, Dietary Restriction, and Exercise Practice among University Students", Journal of Muscle and Joint Health, vol. 20, no. 2, (2013), pp. 151-160.

[22] J. Y. Kim, S. J. Son, J. E. Lee, J. H. Kim and Y. K. Jung, "The Effects of Body Image Satisfaction on Obesity Stress, Weight Control Attitudes, and Eating Disorders among Female Junior High School Students", Family and Environment Research, vol. 47, no. 4, (2009), pp. 49-59.

[23] T. Mase, C. Miyawaki, K. Kouda, Y. Fujita, K. Ohara and H. Nakamura, "Relationship of a Desire of Thinness and Eating Behavior among Japanese Underweight Female Students", Eating and weight disorders, vol. 18, no. 2, (2013), pp. 125-132.

[24] W. H. Ting, C. Y. Huang, Y. K. Tu and K. L. Chien, "Association between Weight Status and Depressive Symptoms in Adolescents: Role of Weight Perception, Weight Concern, and Dietary Restraint”, Eur J Pediatr, vol. 171, no. 8, (2012), pp. 1247-1255.

[25] J. S. Kim and Y. Kim, "Body Perception, Dietary Attitude and Self-Esteem in Middle School Boys and Girls", Korean Home Economics Education Association, vol. 21, no. 1, (2009), pp. 123-139.

[26] J. H. Kang, J. G. Kang, J. H. Kang, S. M. Kim, G. H. Lee and K. M. Choi, "Guidelines for Obesity Treatment 2012", Korean Society for The Study of Obesity, Seoul, (2012).

[27] H. Truby and S. J. Paxton, "Development of The Children's Body Image Scale", The British Journal of Clinical Psychology the British Psychological Society, vol. 41, no. 2, (2002), pp. 185-203.

[28] M. S. Kim and S. Y. Kim, "The Three Dimentions of Eating Behavior", J Korean Acad Nurs, vol. 27, no. 2, (1997), pp. 377-388.

[29] A. J. Stunkard and S. Messick, "The Three-Factor Eating Questionnaire to Measure Dietary Restraint, Disinhibition and Hunger", Journal of psychosomatic research, vol. 29, no. 1, (1985), pp. 71-83.

[30] M. Kovacs, "The Children's Depression, Inventory(CDI)", Psychopharmacol Bull, vol. 21, no, (1985), pp. $995-998$

[31] S. C. Cho and Y. S. Lee, "Development of The Korean form of The Kovacs Children's Depression Inventory", J Korean Neuropsychiatr Assoc, vol. 29, no. 4, (1990), pp. 943-956.

[32] A. P. Pinheiro and E. R. Giugliani, "Body Dissatisfaction in Brazilian Schoolchildren: Prevalence and Associated Factors", Revista de saude publica, vol. 40, no. 3, (2006), pp. 489-496.

[33] G. S. Goldfield, C. Moore, K. Henderson, A. Buchholz, N. Obeid and M. F. Flament, "Body Dissatisfaction, Dietary Restraint, Depression, and Weight Status in Adolescents", Journal of School Health, vol. 80, no. 4, (2010), pp. 186-192.

[34] D. J. Anschutz and R. C. Engels, "The Effects of Playing with Thin Dolls on Body Image and Food Intake in Young Girls", Sex Roles, vol. 63, no. 9-10, (2010), pp. 621-630.

[35] S. Helfert and P. Warschburger, "A Prospective Study on The Impact of Peer and Parental Pressure on Body Dissatisfaction in Adolescent Girls and Boys", Body Image, vol. 8, no. 2, (2011), pp. 101-109.

[36] N. Y. Shin and M. S. Shin, "Body Dissatisfaction, Self-Esteem, and Depression in Obese Korean Children", The Journal of Pediatrics, vol. 152, no. 4, (2008), pp. 502-506.

[37] S. M. Park, E. Y. Kim, Y. I. Rho, S. K. Park and Y. B. Park, "The Relationship between Obesity and Depressive Trends in Elementary School Children", J Korean Pediatr Soc, vol. 45, no. 1, (2002), pp. 1015.

[38] H. J. Lim, "Reserch of Obese Condition of Childhood, Adolescents", National Youth Policy Institude, (2009), pp. 1-137.

[39] E. P. Davila, J. K. Kolodziejczyk, G. J. Norman, K. Calfas, J. S. Huang, C. L. Rock, W. Griswold, J. H. Fowler, S. J. Marshall, A. Gupta and K. Patrick, "Relationships between Depression, Gender, and Unhealthy Weight Loss Practices among Overweight or Obese College Students", Eating Behaviors, vol. 15, no. 2, (2014), pp. 271-274.

[40] E. M. Kim, H. S. Choi and H. Y. Ahn, "The Relationships of Body Image Perception with Cognitive Restraint of Eating and Depression in School-Age Children", Proceedings of the 10th International Workshop on Healthcare and Nursing, Jeju, South Korea, (2016) August 16-19. 


\section{Authors}

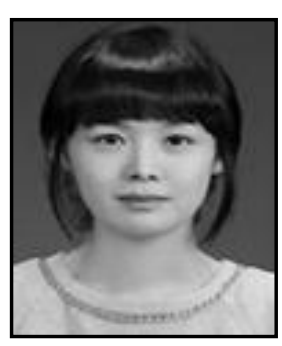

Eun Mi Kim, she is a Doctoral student, College of Nursing, Graduate School, Eulji University.

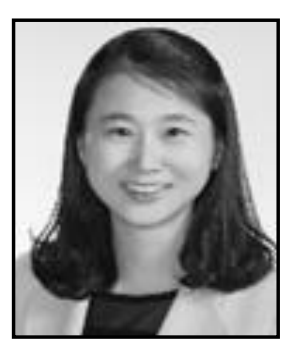

Hye Seon Choi, she is a Professor at College of Nursing, Woosuk University.

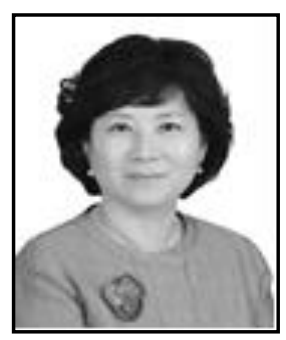

Hye Young Ahn, she is a professor, College of Nursing, Eulji University, Daejeon, Korea. ahanaya@eulji.ac.kr 
International Journal of Bio-Science and Bio-Technology Vol.8, No.6 (2016) 